\title{
Acquired resistance to immunotherapy characterized by bloody pleural effusion and biomarker exploration: a report of 2 cases
}

\author{
Xinyan Zeng", Yiruo Zhang", Yiyin Zhang, Yu Lei, Kangsheng Gu \\ Department of Medical Oncology, The First Affiliated Hospital of Anhui Medical University, Hefei, China \\ "These authors contributed equally to this work and should be considered as co-first authors. \\ Correspondence to: Kangsheng Gu. Department of Medical Oncology, The First Affiliated Hospital of Anhui Medical University, 218 Jixi Road, Hefei \\ 230000, China. Email: gukangsheng@ahmu.edu.cn.
}

\begin{abstract}
Background: With the current wide use of checkpoint inhibitors in the treatment of solid tumors, some patients who initially respond to immunotherapy have been shown to develop acquired resistance. However, the manifestation and underlying mechanisms are currently poorly understood. This is the first reported case of acquired resistance and pleural metastasis with uncontrolled massive bloody pleural effusion after immunotherapy.
\end{abstract}

Case Description: Case 1, a 54-year-old man, was treated with erlotinib for advanced lung adenocarcinoma due to deletion mutations in exon 19 of epidermal growth factor receptor (EGFR). After disease progression, he received pembrolizumab. The best efficacy evaluation during the period was partial response (PR). After 12 cycles of treatment, the patient exhibited rapid disease progression characterized by bloody pleural effusion. Pembrolizumab treatment was discontinued and an attempt was made to inject bevacizumab into the chest cavity; however, the bloody pleural effusion remained difficult to control. The patient experienced a rapid disease progression and died. Case 2, a 71-year-old man, with advanced hepatocellular carcinoma who had previously undergone right hepatectomy, and subsequently received pembrolizumab treatment after disease progression. During the treatment period, his best efficacy evaluation also reached PR. After 23 cycles of treatment, he developed a massive bloody pleural effusion in the chest cavity. He continued to use pembrolizumab for 2 cycles, and anti-angiogenic drugs were injected into the chest cavity, while taking lenvatinib orally; however, the bloody pleural effusion remained difficult to control. The patient eventually died of tumor progression. After the emergence of resistance, we subjected the pleural effusion of the 2 patients to next-generation sequencing (NGS), and preliminarily analyzed the relevant biomarkers.

Conclusions: We found fibroblast growth factor (FGF) family gene mutations in pleural fluid obtained from two patients after immunotherapy resistance. It may suggest that the FGF family may be involved in the development of pembrolizumab resistance. And the combination of fibroblast growth factor receptor (FGFR) inhibitors and immune checkpoint inhibitors (ICIs) may be a promising option for cancer patients.

Keywords: Immunotherapy; acquired resistance; bloody pleural effusion; case report

Submitted Dec 17, 2021. Accepted for publication Feb 21, 2022.

doi: $10.21037 / \mathrm{apm}-22-142$

View this article at: https://dx.doi.org/10.21037/apm-22-142

\section{Introduction}

Monoclonal antibodies against programmed cell death protein-1 (PD-1) receptor on cytotoxic T cells can enhance the anti-tumor immune response in the tumor microenvironment (1). Immune checkpoint inhibitors (ICIs) have been shown to elicit significant therapeutic responses in non-small cell lung cancer (NSCLC), melanoma, liver cancer, and high microsatellite instability (MSI-H) (2-5). Although the mechanism of immunotherapy is different 

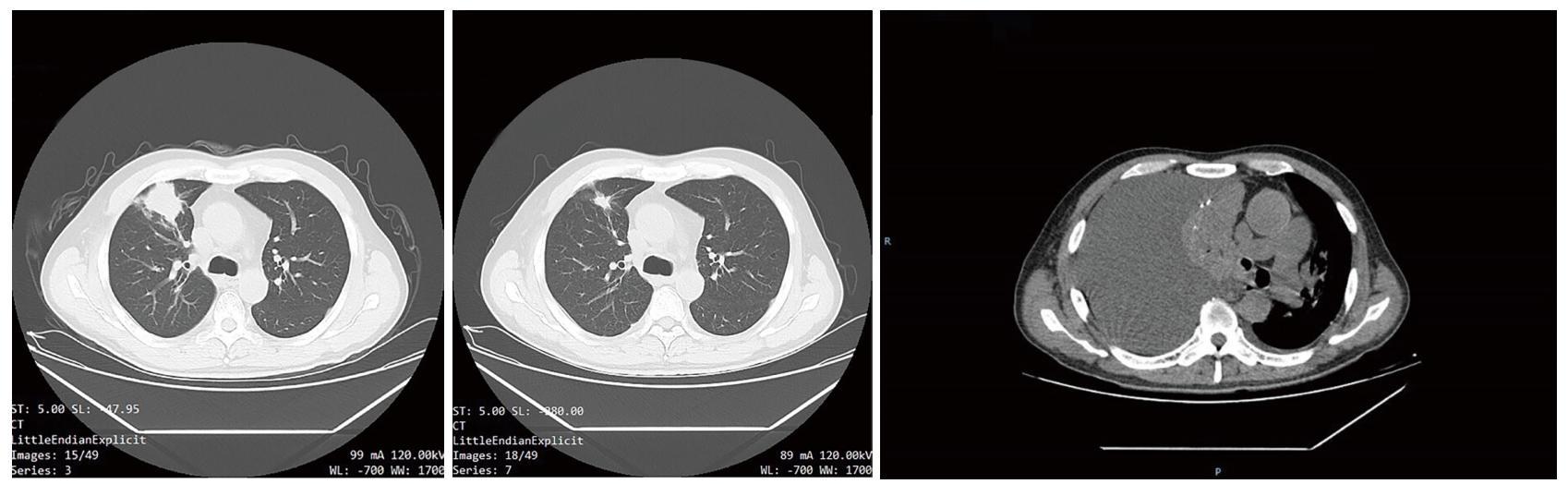

Figure 1 Axial CT images corresponding to the lung lesions in patient 1 before immunotherapy and during immunotherapy, and the pleural effusion that developed after 10 months of immunotherapy. CT, computed tomography.

from traditional chemotherapy and targeted therapy, it also faces the problem of acquired resistance in patients who exhibit an initial response.

The mechanism of acquired resistance in immunotherapy is not clear, and the clinical manifestations are diverse. Herein, we have described a patient with advanced epidermal growth factor receptor (EGFR)-mutant lung adenocarcinoma and a liver cancer patient with lung metastasis who received immunotherapy, subsequently acquired resistance to therapy, and developed metastasis to the pleura, all characterized by uncontrollable massive bloody pleural effusion. When the two patients progressed after first-line treatment, only two immune checkpoint inhibitors (nivolumab and pembrolizumab) were available in China. However, there are more clinical data supporting the efficacy of pembrolizumab in lung cancer and hepatocellular carcinoma (HCC). Therefore, we chose pembrolizumab as the second-line treatment for two patients. We also determined the tumor mutational profile before and after acquired resistance using next-generation sequencing (NGS) in order to provide references for exploring the mechanism of acquired resistance. This is the first case report of acquired resistance with massive bloody pleural effusion after immunotherapy. In addition, we found FGF family gene mutations in pleural fluid obtained from two patients after immunotherapy resistance. This case report suggests that the fibroblast growth factor (FGF) family may be involved in the development of pembrolizumab resistance. We present the following article in accordance with the CARE reporting checklist (available at https://apm.amegroups.com/article/view/10.21037/apm$22-142 / \mathrm{rc})$.

\section{Case presentation}

Patient A, a 54-year-old man with a history of severe smoking (30 packs/year) presented at the hospital due to 4 months of back pain in February 2016. He was diagnosed with right lower lung adenocarcinoma with multiple thoracic vertebral metastases (clinical stage T2aN3M1, IVB stage as per the American Joint Committee on Cancer (AJCC) staging system, 8th edition). No family history was identified. Only exon 19 deletion mutation of the EGFR gene was detected by multigenic NGS. The first-line patients received erlotinib $(150 \mathrm{mg}$, orally, once a day) single drug targeted therapy and achieved stable disease (SD). The progression-free survival (PFS) reached 33 months. In November 2018, he was found to have progressive disease (PD) due to expansion of primary lung lesions, and then a biopsy of right lung lesions was performed again. Genetic testing of EGFR [by amplification-refractory mutation system-polymerase chain reaction (ARMS-PCR)] revealed a deletion mutation in exon 19 of the EGFR gene, which was negative for T790M. Subsequently, the patient received pembrolizumab (200 mg, every 2 weeks) combined with pemetrexed and carboplatin for 6 cycles. The best efficacy evaluation was the PR (Figure 1), and the chemotherapy was stopped due to IV thrombocytopenia. From July 2019, pembrolizumab monotherapy was administered for 6 cycles. Unfortunately, the patient's computed tomography (CT) showed a large amount of effusion in the right pleural cavity and bilateral adrenal involvement in November 2019. The efficacy was evaluated as PD after a PFS of 10 months receiving second-line immunotherapy. The patient's right pleural puncture detected a large amount of bloody pleural effusion and confirmed cancer metastasis. At the same time, 
Table 1 Relevant biomarker detection information

\begin{tabular}{|c|c|c|c|c|c|c|}
\hline \multirow{3}{*}{ No. } & \multicolumn{4}{|c|}{ Clinically significant gene mutation } & \multirow{3}{*}{ TMB } & \multirow{3}{*}{$\begin{array}{c}\text { Microsatellite } \\
\text { instability }\end{array}$} \\
\hline & \multirow{2}{*}{ Common } & \multicolumn{2}{|c|}{ FGF family } & \multirow{2}{*}{ Other } & & \\
\hline & & Gene & Type & & & \\
\hline Patient A & TP53 & FGF19, FGF3, FGF4 & Amplification & EGFR, MET, BRAF, CCND1 & 10.97 mutations/Mb & MSS \\
\hline Patient B & & FGFR3 & p.Arg248Cys & CTNNB1, IDH1 & 0.40 mutations $/ \mathrm{Mb}$ & MSS \\
\hline
\end{tabular}

FGF, fibroblast growth factor; TMB, tumor mutational burden; TP53, tumor protein p53; EGFR, epidermal growth factor receptor; MET, mesenchymal-epithelial transition factor; BRAF, B-Raf proto-oncogene; CCND1, cyclin D1; CTNNB1, Catenin Beta 1; IDH1, isocitrate dehydrogenase 1; MSS, MicroSatellite stability.
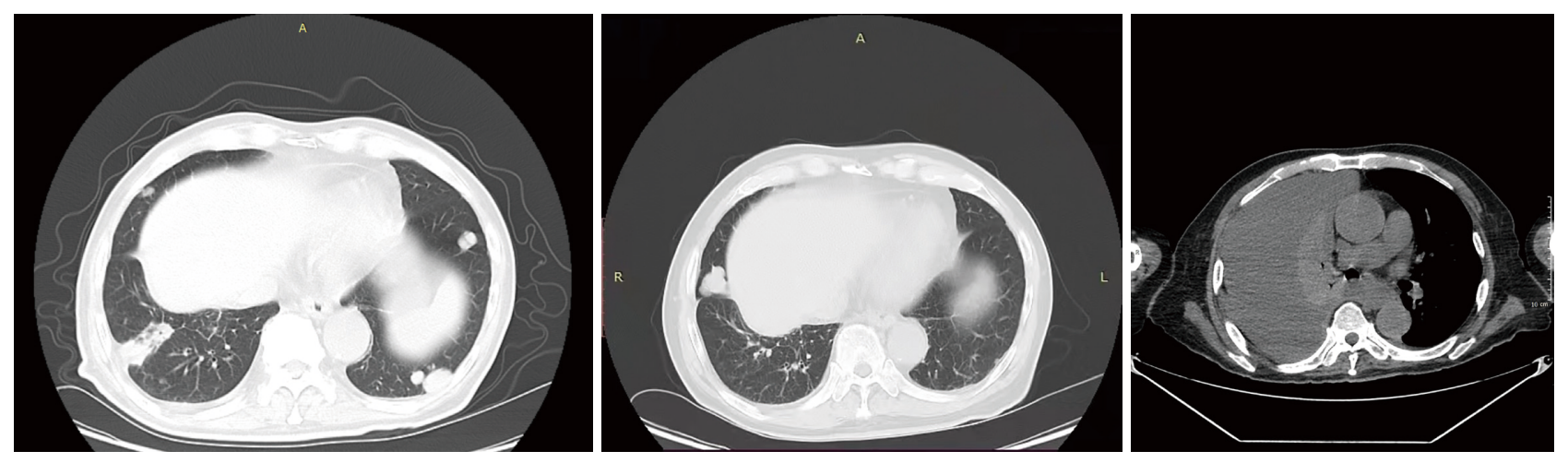

Figure 2 Axial CT images corresponding to the lung metastasis in patient 2 before treatment with immunotherapy and during immunotherapy, and the pleural effusion that developed after 17 months of immunotherapy. CT, computed tomography.

NGS was used for molecular testing of the pleural effusion sample. The relevant biomarker detection information is shown in Table 1. The best supportive treatment was given based on thoracic drainage, and bevacizumab $(300 \mathrm{mg})$ was injected into the thoracic cavity. However, the patient experienced chest tightness, did not improve, and pleural effusion remained uncontrolled. The patient died of tumor progression due to respiratory failure. The time between the occurrence of pleural effusion to death was 32 days.

Patient B was a 68-year-old male with a history of chronic hepatitis B infection. HCC lesion resection had performed on 2 occasions, 26 September 2014, and 28 September 2016. The postoperative pathology confirmed HCC. In November 2016, multiple metastases to the right lung subsequent to liver cancer surgery were found in the follow-up review. The disease progressed after 12 months of oral sorafenib (400 mg, twice a day) treatment. From December 2017, the patient received pembrolizumab monotherapy, and the efficacy was evaluated as PR on 20 August 2018 (Figure 2). By April 2019, after 23 cycles of treatment with pembrolizumab (200 $\mathrm{mg}$, every 2 weeks $)$, the patient experienced chest tightness and a large amount of effusion was detected in the right pleural cavity. Puncture also confirmed a large amount of bloody pleural effusion and the presence of cancer cells. A sample of the pleural effusion was sent for NGS. The relevant biomarker detection information is also shown in Table 1. On the basis of the best supportive treatment, the patient continued to use pembrolizumab for 2 cycles, combined with lenvatinib ( $8 \mathrm{mg}$, orally, once a day), and injection of bevacizumab into the thoracic cavity. The effect was still poor, and the pleural effusion remained uncontrolled. In August 2019, the patient died of respiratory failure due to the continuous deterioration of their condition. The time from the occurrence of pleural effusion to death was 135 days. The timeline of the two patients is shown in Figure 3.

All procedures performed in this study were in accordance with the ethical standards of the institutional and/or national research committee(s) and with the Helsinki Declaration (as revised in 2013). Written informed consent was obtained from both patients for publication of this case report and accompanying images. A copy of the written 


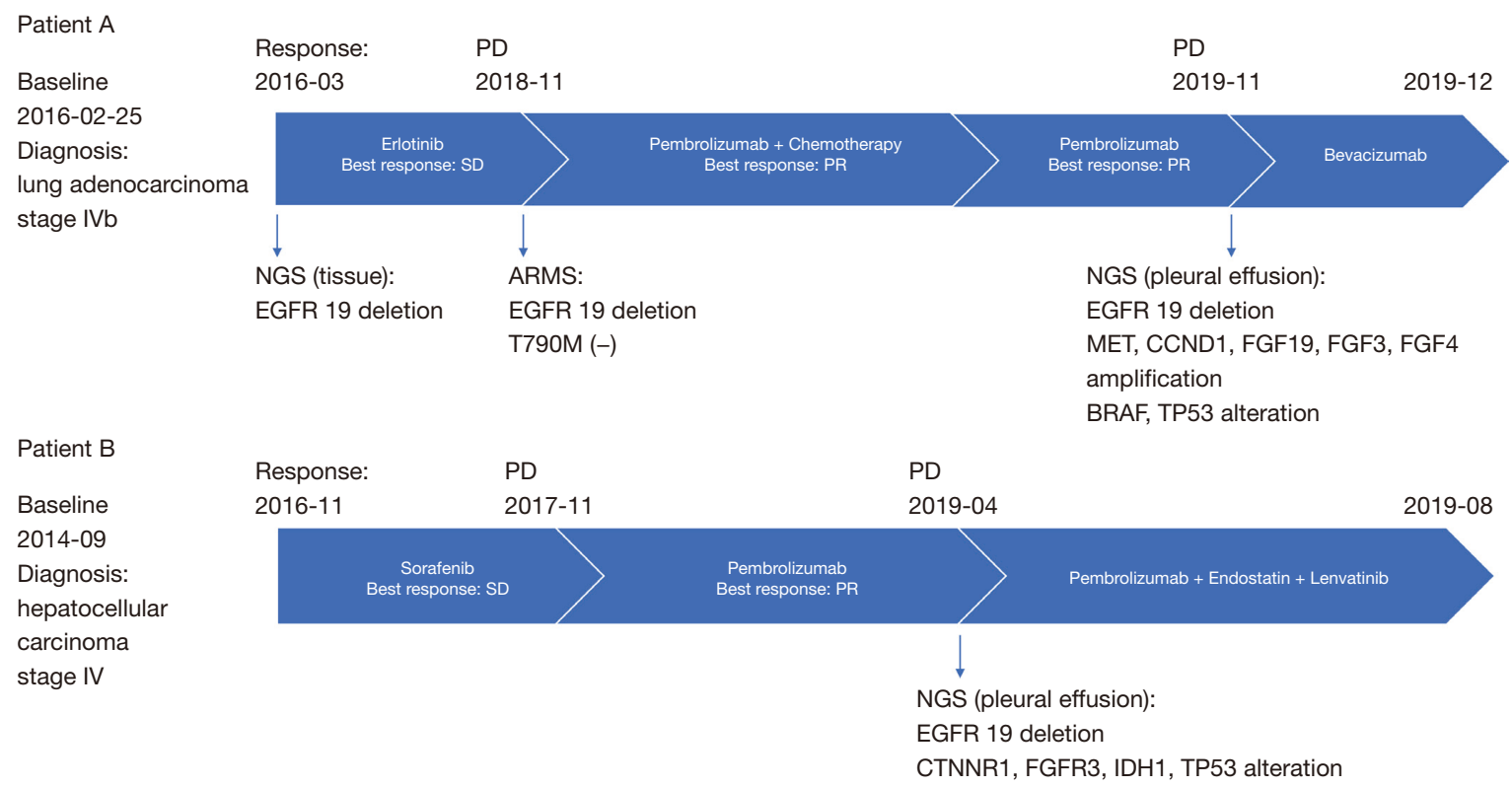

Figure 3 Timeline. PD, progressive disease; SD, stable disease; PR, partial response; NGS, next generation sequencing; ARMS, amplification-refractory mutation system.

consent is available for review by the editorial office of this journal.

\section{Discussion}

Immunotherapy has become an important method in the treatment of malignant tumors; however, similarly to other methods, immunotherapy is susceptible to primary and secondary drug resistance (6). Secondary resistance has been defined as drug use for more than 6 months, and the best response could be complete response (CR), PR, or SD (greater than 6 months), followed by PD (7). The mechanism of tumor resistance is not a simple process of tumor evading the immune system, but the result of interaction among tumor cells, immune microenvironment, and host factors. Tumor internal factors, immune microenvironment, and hostrelated factors may increase immunosuppression and lead to resistance. Known intrinsic factors associated with acquired resistance include loss of target antigen, human leukocyte antigen (HLA), and interferon signaling, as well as loss of $\mathrm{T}$ cell function. The mechanisms of extrinsic resistance in immunotherapy include immune checkpoints, $\mathrm{T}$ cell depletion, and phenotypic changes, immunosuppressive cell population, and release of cytokines and metabolites in the tumor microenvironment (8). In a retrospective analysis of clinical and radiological data of 125 patients with NSCLC treated with anti-PD-1 or programmed death-ligand 1 (PD-L1), the clinical patterns of acquired drug resistance were divided into 2 categories: oligo and systemic types, which were defined as $\leq 2$ disease sites and $\geq 3$ disease sites. Oligoprogressive acquired resistance occurs later than systemic acquired drug resistance, and the survival time after progression is significantly prolonged. The pattern of acquired resistance is usually characterized by oligoprogression, for which locally targeted therapy can improve the survival rate (9). It has also been reported that tumor regression can reoccur after local progression plus radiotherapy (10). A retrospective study of 252 patients with advanced or recurrent NSCLC treated with antiPD-1 antibody in Japan found that after immunotherapy, PFS and overall survival (OS) of patients with malignant pleural effusion (MPE) were significantly shorter than those without MPE (11). The 2 patients we have reported on showed rapid and systematic progress characterized by a large amount of bloody pleural effusion in a short time. The pleural invasion was confirmed by cytology, but it was ineffective in the treatment of local pleural injection of antiangiogenic drugs, and could not receive local treatment such as radiotherapy. The rapid progress of the disease led to the death of the patients.

By comparing the detection results of the NGS gene 
in the pleural effusion of 2 patients, we found that TP53 mutation was present in both cases. One study has shown that TP53 mutation plays a significant role in increasing PD-L1 expression, promoting $\mathrm{T}$ cell infiltration, and enhancing tumor immunogenicity. At the same time, it was confirmed that patients with TP53 mutant lung adenocarcinoma were sensitive to ICI treatment (12).

In addition, mutations in fibroblast growth factor ( $F G F)$ and fibroblast growth factor receptor $(F G F R$ ) family genes were found in these 2 cases. Before treatment, we did not take FGF and FGFR family genes as a routine test, so we could not determine the status of these genes in patients at the beginning. After the emergence of resistance, we obtained pleural effusion from two patients for more comprehensive detection and found the phenomenon of FGF family amplification and mutation. Because these gene changes are rare in the initial state of lung cancer and HCC, we speculated the possible resistance mechanism of the two patients. The FGF family is a fibroblast growth factor and ligand protein of the FGFR family. The FGF/ FGFR signaling pathway can regulate cell proliferation, differentiation, and survival. It plays an important role in early embryonic development, organ formation, angiogenesis, tissue repair, and metabolic regulation. The FGFR gene exerts its activity mainly through the MAPK, tor/Akt, and JAK/STAT signaling pathways, and also participates in the regulation of PD-L1 expression $(13,14)$. At present, abnormal expression of FGFR has been found in a variety of tumors, such as gene amplification, mutation, and fusion gene production. Abnormal FGF signaling can promote tumor development by directly driving cancer cell proliferation and survival and supporting tumor angiogenesis (13). Small sample data have shown that the amplification of 11q13 (CCND1/FGF3/FGF4/ FGF19) region may be related to the excessive progression of ICIs (15). Previously, it was reported that the genomic amplification of the $11 \mathrm{q} 13$ region was related to the poor response of melanoma and esophageal squamous cell carcinoma to toripalimab $(16,17)$. In a phase II clinical trial of toripalimab in the treatment of recurrent or metastatic nasopharyngeal carcinoma (POLARIS-02), patients with $11 \mathrm{q} 13$ genome amplification also did not respond, indicating that there is a potential drug resistance mechanism to immunotherapy (18). In a proteogenomic study on the biology and treatment of human papilloma virus (HPV)-negative head and neck squamous cell carcinoma, it was suggested that $11 \mathrm{q} 13.3$ amplification leads to poor prognosis, which may be a common mechanism by regulating actin dynamics (19). The $F G F R$ inhibitors can target immune cells in the tumor microenvironment and indirectly inhibit PD-L1 expression on tumor cells and stromal/immune cells through the normalization of the tumor microenvironment. Therefore, the combination of FGFR inhibitors and ICIs may be a promising option for cancer patients (20).

There are few clinical reports of pembrolizumab acquired resistance characterized by uncontrolled bloody pleural effusion. We observed 2 similar clinical cases and tried different treatments according to the results of gene detection and related literature. Unfortunately, our research lacked in-depth mechanism exploration, and the treatment had no obvious effect. The patient eventually died due to disease progression. However, based on our analysis of NGS results of pleural effusion sediment wax blocks in both patients, it is suggested that the expansion of the FGF family 11q13 (CCND1/FGF3/FGF4/FGF19) region may show immunotherapeutic resistance and super progress in the form of pleural effusion. We suggest that experts should pay attention to and accumulate FGF family gene status in immunotherapy as much as possible, and explore its value in the efficacy of immunotherapy and hyper progress. For these special forms of immune acquired resistance, we still need to further explore the mechanism and ascertain effective follow-up treatment.

\section{Acknowledgments}

We would like to thank the patients and their families for their consent for publication.

Funding: This study was supported by Wu Jieping Medical Foundation of China (No. 320.6750.2021-02-8).

\section{Footnote}

Reporting Checklist: The authors have completed the CARE reporting checklist. Available at https://apm.amegroups. com/article/view/10.21037/apm-22-142/rc

Conflicts of Interest: All authors have completed the ICMJE uniform disclosure form (available at https://apm. amegroups.com/article/view/10.21037/apm-22-142/coif). The authors have no conflicts of interest to declare.

Ethical Statement: The authors are accountable for all aspects of the work in ensuring that questions related to the accuracy or integrity of any part of the work are 
appropriately investigated and resolved. All procedures performed in this study were in accordance with the ethical standards of the institutional and/or national research committee(s) and with the Helsinki Declaration (as revised in 2013). Written informed consent was obtained from both patients for publication of this case report and accompanying images. A copy of the written consent is available for review by the editorial office of this journal.

Open Access Statement: This is an Open Access article distributed in accordance with the Creative Commons Attribution-NonCommercial-NoDerivs 4.0 International License (CC BY-NC-ND 4.0), which permits the noncommercial replication and distribution of the article with the strict proviso that no changes or edits are made and the original work is properly cited (including links to both the formal publication through the relevant DOI and the license). See: https://creativecommons.org/licenses/by-nc-nd/4.0/.

\section{References}

1. Gangadhar TC, Salama AK. Clinical applications of PD1-based therapy: a focus on pembrolizumab (MK-3475) in the management of melanoma and other tumor types. Onco Targets Ther 2015;8:929-37.

2. Mok TSK, Wu YL, Kudaba I, et al. Pembrolizumab versus chemotherapy for previously untreated, PD-L1expressing, locally advanced or metastatic non-small-cell lung cancer (KEYNOTE-042): a randomised, open-label, controlled, phase 3 trial. Lancet 2019;393:1819-30.

3. Robert C, Ribas A, Schachter J, et al. Pembrolizumab versus ipilimumab in advanced melanoma (KEYNOTE-006): post-hoc 5-year results from an openlabel, multicentre, randomised, controlled, phase 3 study. Lancet Oncol 2019;20:1239-51.

4. Zhu AX, Finn RS, Edeline J, et al. Pembrolizumab in patients with advanced hepatocellular carcinoma previously treated with sorafenib (KEYNOTE-224): a non-randomised, open-label phase 2 trial. Lancet Oncol 2018;19:940-52. Erratum in: Lancet Oncol 2018;19:e440.

5. Marabelle A, Fakih M, Lopez J, et al. Association of tumour mutational burden with outcomes in patients with advanced solid tumours treated with pembrolizumab: prospective biomarker analysis of the multicohort, openlabel, phase 2 KEYNOTE-158 study. Lancet Oncol 2020;21:1353-65.

6. Restifo NP, Smyth MJ, Snyder A. Acquired resistance to immunotherapy and future challenges. Nat Rev Cancer
2016;16:121-6.

7. Kluger HM, Tawbi HA, Ascierto ML, et al. Defining tumor resistance to PD-1 pathway blockade: recommendations from the first meeting of the SITC Immunotherapy Resistance Taskforce. J Immunother Cancer 2020;8:e000398.

8. Sharma P, Hu-Lieskovan S, Wargo JA, et al. Primary, Adaptive, and Acquired Resistance to Cancer Immunotherapy. Cell 2017;168:707-23.

9. Heo JY, Yoo SH, Suh KJ, et al. Clinical pattern of failure after a durable response to immune check inhibitors in non-small cell lung cancer patients. Sci Rep 2021;11:2514.

10. Herrera FG, Bourhis J, Coukos G. Radiotherapy combination opportunities leveraging immunity for the next oncology practice. CA Cancer J Clin 2017;67:65-85.

11. Shibaki R, Murakami S, Shinno Y, et al. Malignant pleural effusion as a predictor of the efficacy of anti-PD-1 antibody in patients with non-small cell lung cancer. Thorac Cancer 2019;10:815-22.

12. Dong ZY, Zhong WZ, Zhang XC, et al. Potential Predictive Value of TP53 and KRAS Mutation Status for Response to PD-1 Blockade Immunotherapy in Lung Adenocarcinoma. Clin Cancer Res 2017;23:3012-24.

13. Babina IS, Turner NC. Advances and challenges in targeting FGFR signalling in cancer. Nat Rev Cancer 2017;17:318-32.

14. Chen J, Jiang CC, Jin L, et al. Regulation of PD-L1: a novel role of pro-survival signalling in cancer. Ann Oncol 2016;27:409-16.

15. Singavi AK, Menon S, D Kilari, et al. Predictive biomarkers for hyper-progression (HP) in response to immune checkpoint inhibitors (ICI) - analysis of somatic alterations (SAs). Ann Oncol 2017;28:V405.

16. Tang B, Chi Z, Chen Y, et al. Safety, Efficacy, and Biomarker Analysis of Toripalimab in Previously Treated Advanced Melanoma: Results of the POLARIS-01 Multicenter Phase II Trial. Clin Cancer Res 2020;26:4250-9.

17. Wang F, Ren C, Zhao Q, et al. Association of frequent amplification of chromosome 11q13 in esophageal squamous cell cancer with clinical benefit to immune check point blockade. J Clin Oncol 2019;37:abstr 4036.

18. Wang FH, Wei XL, Feng J, et al. Efficacy, Safety, and Correlative Biomarkers of Toripalimab in Previously Treated Recurrent or Metastatic Nasopharyngeal Carcinoma: A Phase II Clinical Trial (POLARIS-02). J Clin Oncol 2021;39:704-12.

19. Huang C, Chen L, Savage SR, et al. Proteogenomic 
insights into the biology and treatment of HPV-negative head and neck squamous cell carcinoma. Cancer Cell 2021;39:361-79.e16.

20. Palakurthi S, Kuraguchi M, Zacharek SJ, et al. The Combined Effect of FGFR Inhibition and PD-1 Blockade

Cite this article as: Zeng X, Zhang Y, Zhang Y, Lei Y, Gu K. Acquired resistance to immunotherapy characterized by bloody pleural effusion and biomarker exploration: a report of 2 cases. Ann Palliat Med 2022;11(2):843-849. doi: 10.21037/apm-22-142
Promotes Tumor-Intrinsic Induction of Antitumor Immunity. Cancer Immunol Res 2019;7:1457-71.

(English Language Editor: J. Jones) 